\title{
ATENCIÓN DE ENFERMERÍA A PACIENTE CON PIE DIABÉTICO
}

\section{ARTÍCULO ORIGINAL}

CUNHA, Amanda Priscilla da ${ }^{1}$

CUNHA, Amanda Priscilla da. Atención de enfermería a paciente con pie diabético. Revista Científica Multidisciplinar Núcleo do Conhecimento. Año. 06, Ed. 12, Vol. 12, págs. 111-126. Diciembre 2021. ISSN: 2448-0959, Enlace de acceso: https://www.nucleodoconhecimento.com.br/salud/pie-diabetico,

DOI: 10.32749/nucleodoconhecimento.com.br/salud/pie-diabetico

\section{RESUMEN}

Introducción: El pie diabético también llamado por un estado fisiopatológico multifacético se caracteriza por lesiones que surgen de los pies de la persona con diabetes sin el tratamiento adecuado y los cuidados específicos. Se estima que la incidencia de úlcera del pie diabético alcanza el 6,3\% de los pacientes diabéticos y su prevalencia se acerca al $10 \%$, y las personas con bajo nivel socioeconómico son las mayores víctimas. En vista de esta evidencia, el trabajo se basa en la siguiente pregunta problemática: ¿Qué cuidados desarrollan las enfermeras en pacientes con pies diabéticos? Objetivo general: Evidenciar el cuidado del pie diabético relacionado con la práctica de las enfermeras. Metodología: Se realizó una revisión integradora en la Base de Datos Bibliográfica Especializada en Enfermería (BDENF), Literatura Latinoamericana y del Caribe en Ciencias de la Salud (LILACS) y la Scientific Electronic Library Online (SCIELO) y que dio como resultado siete artículos. Resultados: Los estudios mostraron cómo el cuidado de las enfermeras con pie diabético educación para la salud, educación continua y permanente, técnicas de evaluación al pie diabético que implican exámenes físicos y autocuidado de pacientes con pie diabético. Se refuerza que este cuidado debe ser realizado de manera prioritaria por las enfermeras, ya que las enfermeras tienen una atención

\footnotetext{
${ }^{1}$ Máster en Gestión Hospitalaria y Sistemas de Salud, Enfermera Licenciada en Enfermería. ORCID: 000-00015456-1848.
}

RC: 105163

Disponible: https://www.nucleodoconhecimento.com.br/salud/pie-diabetico 
específica y calificada al paciente en sus principales atribuciones. Conclusión: Se concluye, por lo tanto, que el estudio identificó varias formas de atención proporcionadas por las enfermeras y que todas estas formas son importantes para la continuidad del tratamiento y la prevención de complicaciones, sin embargo, depende de la enfermera ser un profesional que valore la calidad de su atención en base a conceptos teóricos que conduzcan a una formación frecuente.

Palabras clave: Atención al paciente, Diabetes Mellitus, Pie diabético.

\section{INTRODUCCIÓN}

La Diabetes Mellitus (DM) es una enfermedad desarrollada en el sistema endocrino y/o un trastorno metabólico crónico, que representa un grave problema de salud a nivel mundial y tiene como principal característica la hiperglucemia, alcanzando un nivel de glucosa en sangre superior a $126 \mathrm{mg} / \mathrm{dL}$ en ayunas $0>200 \mathrm{mg} / \mathrm{dL}$ en la etapa postprandial, y que puede haberse asociado con resistencia a la insulina, y que puede haberse asociado con resistencia a la insulina, secreción inadecuada de insulina o secreción excesiva de glucagón (ASCHNER et al., 2016).

Por lo tanto, la hiperglucemia causa incapacidad total o parcial de la producción de insulina, según la Sociedad Brasileña de Diabetes (SBD), la American Diabetes Association (ADA) y la Organización Mundial de la Salud (OMS) La DM se divide en cuatro grupos: diabetes mellitus tipo 1, diabetes mellitus tipo 2, diabetes mellitus gestacional y otros tipos específicos de diabetes (OMS, 1999; SBD, 2017; ADA 2017).

SBD describe los factores de riesgo para la DM, que se citan como los siguientes: diagnóstico de prediabetes - disminución de la tolerancia a la glucosa o alteración de la glucosa en ayunas; hipertensión; hipercolesterolemia o cambios en la tasa de triglicéridos en la sangre; sobrepeso y obesidad, especialmente si la grasa se concentra alrededor de la cintura; antecedentes familiares; bebé que pesa más de cuatro kilos o diabetes gestacional; síndrome de ovario poliquístico; diagnóstico de 
algunos trastornos psiquiátricos, como esquizofrenia, depresión, trastorno bipolar y uso de medicamentos psicotrópicos; otros (SBD, 2018).

Por tratarse de una enfermedad que afecta aproximadamente al 3\% de la población mundial y con una prevalencia aproximada del $6,2 \%$ de la población en Brasil, cuya prevalencia aumenta debido al envejecimiento poblacional (MUZY et al., 2021), la DM ha causado una carga en las arcas públicas, además de permitir también altos costos para realizar el tratamiento de sus complicaciones como: pie diabético, enfermedades cardiovasculares, amputación y otras. Cabe mencionar que el tratamiento farmacológico ha ido avanzando en el control de la DM, sin embargo, la educación para la salud como forma de prevención debe ser transmitida a la población en general de manera individual o grupal, haciendo énfasis en la problemática de la enfermedad y sus daños a la salud ( SILVA, 2018). ).

En cuanto a las complicaciones de la DM, el pie diabético también se denomina por su estado fisiopatológico multifacético, caracterizado por lesiones que surgen de los pies de la persona con diabetes por neuropatía en el $90 \%$ de los casos, en los que predominan la enfermedad vascular periférica y las deformidades (CUBAS et al., 2017).

De Sousa Mendonca; Moraes; Moura (2017) describe que las lesiones del pie diabético existentes provienen de la combinación de dos o más factores de riesgo que pueden estar relacionados con traumatismos intrínsecos o extrínsecos, asociados a neuropatía periférica provocando pérdida de sensibilidad, facilitando el riesgo de cortes; enfermedad vascular periférica resultante de la reducción del flujo sanguíneo en las extremidades inferiores y cambios biomecánicos que ocurren cuando se restringe el movimiento de las articulaciones del pie y el tobillo.

Se estima que la incidencia de úlcera del pie diabético alcanza el 6,3\% de los pacientes diabéticos y su prevalencia se acerca al $10 \%$, y las personas con bajo nivel socioeconómico son las mayores víctimas. La amputación del pie diabético es otro problema y que a nivel mundial cada tres minutos a una persona se le amputó la extremidad debido a la DM (ARRUDA et al., 2021). 
El buen manejo de los factores que intervienen en la etiología de la lesión existente en el pie diabético se hace efectivo cuando es realizado por la enfermera, por lo que la enfermera debe estar entrenado y cualificada para realizar la consulta de enfermería con el fin de no detectar cambios dermatológicos, musculoesqueléticos, vasculares y neurológicos en el pie diabético (FÉLIX et al., 2021).

Por lo tanto, el cuidado de los pies diabéticos cobra mucha importancia dentro de la salud pública y la enfermera, como profesional que tiene en su premisa el cuidado del paciente, debe estar atenta y cualificada respecto a las nuevas formas de tratamiento para reducir las amputaciones. Ante este escenario de tasas alarmantes, es fundamental observar cómo se sitúan las enfermeras en las prácticas en el cuidado de los pies diabéticos.

Ante esta evidencia que involucra números alarmantes en relación al pie diabético, el trabajo se basa en la siguiente pregunta problemática: ¿Qué cuidados desarrollan las enfermeras en pacientes con pie diabético?

El estudio en cuestión tiene como objetivo evidenciar el cuidado del pie diabético relacionado con la práctica de las enfermeras.

\section{METODOLOGÍA}

Para este estudio, se realizó una revisión integradora de la literatura. Según Mendes; Silveira y Galvão (2019), el método de revisión integrativa se desarrolla siguiendo pasos bien definidos; son: identificación del tema y selección de la cuestión del norte; establecer criterios de inclusión y exclusión; identificación de estudios preseleccionados y seleccionados; categorización de estudios seleccionados; análisis e interpretación de los resultados y presentación del resumen de la revisión.

El primer paso de la revisión integradora es la cuestión septentrional, que conduce al tema y los objetivos enumerados. Por lo tanto, la pregunta que surge en este artículo es: ¿Qué cuidados desarrollan las enfermeras en pacientes con pies diabéticos? 
Para establecer los criterios de inclusión se utilizaron: artículos publicados y disponibles para su lectura, ya que el sujeto refleja a la enfermera en sus atribuciones, se eligió la Base de Datos de BDENF, LILACS y SCIELO, la delimitación temporal fue en los años 2016 a 2020, es decir, en los últimos cinco años, y se eligió el idioma portugués para esta inclusión.

Para los criterios de exclusión se eliminaron: artículos que tienen duplicidad, artículos incompletos, annais, textos de opinión, editoriales y cartas al lector, artículos de revisión, tesis, disertaciones, textos completos, pero que no se relacionan con el tema.

El período de recolección de datos correspondió al mes de septiembre de 2021. Los descriptores nacionales identificados fueron: "cuidados de enfermería", "pie diabético", "enfermera", "diabetes mellitus". El operador booleano and se utilizó para asociar los descriptores en la estrategia de búsqueda en la plataforma de base de datos.

Para la selección de estudios seleccionados y preseleccionados, se realizó una lectura exhaustiva de los títulos y resúmenes de los artículos encontrados, y luego una lectura más completa a lo largo del artículo, buscando en sus resultados la identificación para lograr el objetivo propuesto en este artículo.

Se realizaron dos búsquedas, la primera utilizó la siguiente estrategia de búsqueda: cuidados de enfermería and pie diabético and diabetes mellitus, con esta estrategia se encontraron 186 artículos. Después de utilizar los filtros: texto completo y disponible, base de datos, idioma portugués y delimitación temporal de los últimos cinco años, quedaban 24 artículos para la elegibilidad. La segunda búsqueda se realizó con la siguiente estrategia: enfermera and pie diabético encontrando 95 artículos. Después de usar los filtros, 11 artículos permanecieron para la elegibilidad.

Los estudios fueron categorizados a partir de una imagen sinóptica y el análisis y presentación de los resultados se realizó después de la categorización que identificó 
y definió los núcleos de significado de los estudios, y que a partir del análisis temático de Minayo (2012), hubo discusión entre los autores encontrados.

Para la síntesis del conocimiento, se realizó un análisis de las limitaciones, representatividad del estudio para la comunidad en general (relevancia) y estudios futuros identificados en el curso de este artículo. Todas las producciones utilizadas en este estudio están rigurosamente referenciadas de acuerdo con los estándares de la Asociación Brasileña de Normas Técnicas (ABNT).

\section{RESULTADOS Y DISCUSIONES}

Los artículos restantes para la elegibilidad con ambas estrategias totalizaron 35 artículos. Inicialmente, se eliminaron artículos de revisión, trabajos de finalización de cursos, cartas al lector, informes de experiencias, estudios de casos, artículos duplicados y que en sus títulos, resúmenes y lectura completa no trajeron a la enfermera y su cuidado como tema al tema, dejando así 07 artículos para esta revisión.

Para una mejor ilustración de los resultados encontrados, la siguiente tabla incluirá la caracterización de los estudios seleccionados. Los datos se refieren a: título del artículo, año de publicación, base de datos, revista, ubicación, objetivo y resultados.

Tabla 1. Artículos encontrados en la base de datos BDENF, LILACS y SCIELO sobre cuidados de enfermería para pacientes con pie diabético.

\begin{tabular}{|c|c|c|c|c|c|}
\hline $\begin{array}{l}\text { Título/ Año de } \\
\text { publicación }\end{array}$ & Base & $\begin{array}{l}\text { periódic } \\
0\end{array}$ & Gol & Local & Resultados \\
\hline Conheciment & BDENF & $\begin{array}{l}\text { Rev. } \\
\text { enferm }\end{array}$ & Comprender el & $\begin{array}{l}\text { Estrategia } \\
\text { de Salud }\end{array}$ & $\begin{array}{l}\text { Realizar } \\
\text { periódicamente }\end{array}$ \\
\hline $\begin{array}{l}\text { enfermeiro } \\
\text { acerca dos } \\
\text { cuidados com } \\
\text { o pé diabético }\end{array}$ & & $\begin{array}{l}\text { enterm. } \\
\text { UFPE } \\
\text { on line }\end{array}$ & $\begin{array}{l}\text { conocimiento } \\
\text { de la } \\
\text { enfermera } \\
\text { sobre el } \\
\text { cuidado del pie }\end{array}$ & $\begin{array}{l}\text { de Salud } \\
\text { de la } \\
\text { Familia/ES } \\
\text { F }\end{array}$ & $\begin{array}{l}\text { periodicamente } \\
\text { un examen } \\
\text { físico de los } \\
\text { pies en } \\
\text { diabéticos; }\end{array}$ \\
\hline
\end{tabular}




\begin{tabular}{|c|c|c|c|c|c|}
\hline 2019 & & & $\begin{array}{l}\text { diabético en } \\
\text { Atención } \\
\text { Primaria }\end{array}$ & & $\begin{array}{l}\text { Uso de } \\
\text { instrumentos } \\
\text { para la } \\
\text { evaluación del } \\
\text { pie diabético } \\
\text { Autocuidado a } \\
\text { los pies }\end{array}$ \\
\hline $\begin{array}{l}\text { Riscos } \\
\text { associados à } \\
\text { mortalidade } \\
\text { em pacientes } \\
\text { atendidos em } \\
\text { um programa } \\
\text { de prevenção } \\
\text { do pé } \\
\text { diabético } \\
2018\end{array}$ & $\begin{array}{l}\text { LILACS } \\
/ \\
\text { BDENF }\end{array}$ & $\begin{array}{l}\text { Rev. } \\
\text { gaúch. } \\
\text { enferm }\end{array}$ & $\begin{array}{l}\text { Identificar en } \\
\text { pacientes con } \\
\text { diabetes tipo } 2 \\
\text { qué cambios } \\
\text { en los pies } \\
\text { estarían } \\
\text { asociados con } \\
\text { características } \\
\text { demográficas, } \\
\text { clínicas, } \\
\text { bioquímicas y } \\
\text { de tratamiento } \\
\text { y cuáles de } \\
\text { ellos aumentan } \\
\text { el riesgo de } \\
\text { mortalidad }\end{array}$ & $\begin{array}{l}\text { Programa } \\
\text { educativo } \\
\text { para la } \\
\text { prevención } \\
\text { y } \\
\text { tratamiento } \\
\text { del pie } \\
\text { diabético } \\
\text { en un } \\
\text { hospital } \\
\text { general y } \\
\text { universitari } \\
\text { o de alta } \\
\text { complejida } \\
\text { d }\end{array}$ & $\begin{array}{l}\text { Educación } \\
\text { sanitaria sobre } \\
\text { higiene de los } \\
\text { pies, la } \\
\text { elección del } \\
\text { calzado } \\
\text { adecuado, la } \\
\text { mejor conducta } \\
\text { en situaciones } \\
\text { de } \\
\text { emergencia. } \\
\text { Cuidados } \\
\text { sistémicos } \\
\text { como no } \\
\text { fumar, } \\
\text { mantener un } \\
\text { buen control } \\
\text { glucémico, } \\
\text { controlar las } \\
\text { tasas de } \\
\text { lípidos en } \\
\text { sangre y el uso } \\
\text { correcto de los } \\
\text { medicamentos }\end{array}$ \\
\hline
\end{tabular}




\begin{tabular}{|c|c|c|c|c|c|}
\hline $\begin{array}{l}\text { Condutas dos } \\
\text { enfermeiros } \\
\text { da atenção } \\
\text { primária no } \\
\text { cuidado a } \\
\text { pessoas com } \\
\text { pé diabético } \\
2017\end{array}$ & BDENF & $\begin{array}{l}\text { Rev. } \\
\text { enferm. } \\
\text { UFPE } \\
\text { on line }\end{array}$ & $\begin{array}{l}\text { Conocer las } \\
\text { actuaciones de } \\
\text { las enfermeras } \\
\text { de atención } \\
\text { primaria en la } \\
\text { atención de } \\
\text { personas con } \\
\text { Diabetes } \\
\text { Mellitus (DM) } \\
\text { relacionadas } \\
\text { con el pie } \\
\text { diabético }\end{array}$ & $\begin{array}{l}\text { Estrategia } \\
\text { de Salud } \\
\text { de la } \\
\text { Familia }\end{array}$ & $\begin{array}{l}\text { Frecuencia y } \\
\text { programación } \\
\text { de consultas, } \\
\text { dinámica de } \\
\text { servicio, } \\
\text { criterios para el } \\
\text { examen de los } \\
\text { pies, } \\
\text { autocuidado }\end{array}$ \\
\hline $\begin{array}{l}\text { Ações do } \\
\text { enfermeiro na } \\
\text { prevenção do } \\
\text { pé diabético: } \\
0 \text { olhar da } \\
\text { pessoa com } \\
\text { diabetes } \\
\text { mellitus } \\
2017\end{array}$ & $\begin{array}{l}\text { LILACS } \\
/ \\
\text { BDENF }\end{array}$ & $\begin{array}{l}\text { Rev. } \\
\text { Pesqui. } \\
\text { (Univ. } \\
\text { Fed. } \\
\text { Estado } \\
\text { Rio J., } \\
\text { Online) }\end{array}$ & $\begin{array}{l}\text { Investigar las } \\
\text { acciones } \\
\text { realizadas por } \\
\text { las enfermeras } \\
\text { en la } \\
\text { prevención del } \\
\text { pie diabético } \\
\text { desde la } \\
\text { perspectiva de } \\
\text { la persona con } \\
\text { DM. }\end{array}$ & $\begin{array}{l}\text { Proyecto } \\
\text { de } \\
\text { extensión } \\
\text { universitari } \\
\text { a }\end{array}$ & $\begin{array}{l}\text { Acciones } \\
\text { específicas, } \\
\text { que involucran } \\
\text { adecuadament } \\
\text { e el examen de } \\
\text { los pies, el } \\
\text { diálogo } \\
\text { comunicativo, } \\
\text { la educación } \\
\text { para la salud. }\end{array}$ \\
\hline $\begin{array}{l}\text { Alterações } \\
\text { nos pés do } \\
\text { idoso } \\
\text { hospitalizado: } \\
\text { um olhar } \\
\text { cuidadoso da } \\
\text { enfermagem } \\
2017\end{array}$ & $\begin{array}{l}\text { LILACS } \\
/ \\
\text { BDENF }\end{array}$ & $\begin{array}{l}\text { Esc. } \\
\text { Anna } \\
\text { Nery } \\
\text { Rev. } \\
\text { Enferm }\end{array}$ & $\begin{array}{l}\text { Caracterizar el } \\
\text { perfil } \\
\text { podológico de } \\
\text { ancianos } \\
\text { internados en } \\
\text { las salas de un } \\
\text { hospital } \\
\text { universitario; }\end{array}$ & Hospital & $\begin{array}{l}\text { Fortalecimiento } \\
\text { de los vínculos } \\
\text { con estos } \\
\text { pacientes } \\
\text { diabéticos para } \\
\text { que acudan al } \\
\text { puesto a } \\
\text { realizar }\end{array}$ \\
\hline
\end{tabular}




\begin{tabular}{|c|c|c|c|c|c|}
\hline & & & $\begin{array}{l}\text { identificar las } \\
\text { demandas de } \\
\text { cuidado de los } \\
\text { pies de los } \\
\text { pacientes } \\
\text { ancianos } \\
\text { hospitalizados; } \\
\text { y analizar las } \\
\text { posibilidades } \\
\text { de las acciones } \\
\text { de las } \\
\text { enfermeras } \\
\text { con estos } \\
\text { ancianos. }\end{array}$ & & $\begin{array}{l}\text { exámenes de } \\
\text { pies con } \\
\text { frecuencia } \\
\text { Formación y } \\
\text { cualificación } \\
\text { para atender } \\
\text { estos } \\
\text { problemas, } \\
\text { evitando } \\
\text { mayores } \\
\text { complicaciones } \\
\text { y reduciendo } \\
\text { las futuras } \\
\text { demandas } \\
\text { asistenciales }\end{array}$ \\
\hline $\begin{array}{l}\text { Atuação dos } \\
\text { enfermeiros } \\
\text { da estratégia } \\
\text { saúde da } \\
\text { família na } \\
\text { prevenção do } \\
\text { pé diabético } \\
2016\end{array}$ & $\begin{array}{l}\text { LILACS } \\
/ \\
\text { BDENF }\end{array}$ & $\begin{array}{l}\text { Rev. } \\
\text { Pesqui. } \\
\text { (Univ. } \\
\text { Fed. } \\
\text { Estado } \\
\text { Rio J., } \\
\text { Online) ; }\end{array}$ & $\begin{array}{l}\text { Identificar la } \\
\text { orientación } \\
\text { proporcionada } \\
\text { por las } \\
\text { enfermeras a } \\
\text { las personas } \\
\text { con DM en el } \\
\text { cuidado de los } \\
\text { pies; Investigar } \\
\text { la frecuencia } \\
\text { del examen del } \\
\text { pie y los } \\
\text { aspectos } \\
\text { evaluados; } \\
\text { para verificar } \\
\text { qué }\end{array}$ & $\begin{array}{l}\text { Estrategia } \\
\text { de Salud } \\
\text { de la } \\
\text { Familia }\end{array}$ & $\begin{array}{l}\text { Educación } \\
\text { para la salud } \\
\text { de los } \\
\text { diabéticos, } \\
\text { examen de los } \\
\text { pies de } \\
\text { personas con } \\
\text { DM, formación } \\
\text { de grupos de } \\
\text { convivencia, } \\
\text { apósitos en } \\
\text { técnicas } \\
\text { adecuadas al } \\
\text { tipo de lesión } \\
\text { del pie. }\end{array}$ \\
\hline
\end{tabular}




\begin{tabular}{|c|c|c|c|c|c|}
\hline & & & $\begin{array}{l}\text { actividades de } \\
\text { educación para } \\
\text { la salud } \\
\text { realizan las } \\
\text { enfermeras } \\
\text { para las } \\
\text { personas con } \\
\text { DM. }\end{array}$ & & \\
\hline $\begin{array}{l}\text { A perspectiva } \\
\text { da } \\
\text { vulnerabilidad } \\
\text { e na } \\
\text { avaliação do } \\
\text { pé diabético } \\
\text { sob a ótica de } \\
\text { enfermeiros } \\
2016\end{array}$ & BDENF & $\begin{array}{l}\text { Cogitare } \\
\text { enferm }\end{array}$ & $\begin{array}{l}\text { Escribir las } \\
\text { condiciones } \\
\text { determinantes } \\
\text { sociales en } \\
\text { salud, } \\
\text { identificadas } \\
\text { por } \\
\text { enfermeras } \\
\text { como } \\
\text { relevantes para } \\
\text { el } \\
\text { establecimient } \\
\text { o } \\
\text { vulnerabilidade } \\
\text { s de la persona } \\
\text { con Diabetes } \\
\text { Mellitus. }\end{array}$ & $\begin{array}{l}\text { Enfermeras } \\
\text { elegidas al } \\
\text { azar en } \\
\text { Curitiba }\end{array}$ & $\begin{array}{l}\text { Estimular al } \\
\text { diabético para } \\
\text { que vaya a una } \\
\text { evaluación } \\
\text { nutricional y } \\
\text { siga la dieta. }\end{array}$ \\
\hline
\end{tabular}

Fuente: Autor.

El estudio en sus objetivos menciona tres tipos de cuidados realizados por enfermeras para pacientes con pie diabético: El primero que más surgió se basa en acciones dirigidas a la educación para la salud como principio fundamental de la prevención de enfermedades y sus complicaciones, el segundo cuidado se basa en 
técnicas de evaluación del pie en diabéticos como forma de surgir de complicaciones $y$ el tercer cuidado se centra en las enfermeras como estimulador y motivador. pacientes diabéticos a su autocuidado con los pies. Estas tres precauciones se discutirán en sus temas de acuerdo con los estudios seleccionados.

\subsection{LA EDUCACIÓN PARA LA SALUD COMO FORMA DE PREVENCIÓN}

Por lo tanto, desde la educación para la salud, el paciente puede comprender las razones por las que el proceso de cicatrización de heridas es lento, las razones de las principales complicaciones y especialmente los conocimientos relacionados con la prevención de riesgos. Las enfermeras deben ser capaces de reconocer las necesidades del paciente y así contribuir a diagnósticos de enfermería precisos y seguros relacionados con el herido en tu estado.

Scain; Franzen; Hirakata (2018) informa que las enfermeras han estado manteniendo esfuerzos para realizar educación para la salud con los pacientes, volviéndose persistentes e insistentes para continuar el proceso educativo en pacientes con pies diabéticos, usar la creatividad con el desarrollo de tecnologías educativas como carpetas, videos, así como estar disponibles para hacer preguntas sobre alguna condición anormal con el paciente desde tecnologías de redes sociales como Whatsapp / teléfono.

Camillo et al. (2016) menciona que el concepto de educación para la salud va más allá de traer datos o incluso eliminar las dudas que surgen, los autores consideran la educación para la salud como un proceso sistemático, continuo y permanente, dirigido a la formación y desarrollo de la conciencia crítica del ciudadano, a través de la búsqueda de soluciones colectivas a los problemas experimentados y su "participación real" en el ejercicio del control social.

Vargas et al. (2017) trae la educación para la salud como uno de los principales propósitos de las atribuciones de las enfermeras al cuidado, los autores refuerzan que la educación para la salud favorece al paciente una postura más proactiva, 
demostrando que el paciente debe tener seguridad sobre el cuidado del pie diabético.

La autonomía y la seguridad de la atención también se señalaron como resultados de la educación para la salud realizada por las enfermeras en el estudio de Pereira et al. (2017), los autores informan que la educación para la salud se convierte en el principal mecanismo de prevención contra las enfermedades y sus complicaciones, en relación con el pie diabético, la educación para la salud promueve la seguridad, la confianza y la autonomía del paciente.

La demostración de que la educación para la salud es efectiva se identificó en un estudio descriptivo de enfoque cuantitativo en el que se demostró que la educación para la salud aportaba a los pacientes un conocimiento teórico sobre la enfermedad respetando la cultura y creencia de los pacientes, y que la promoción de los procesos educativos se lleva a cabo no solo de manera conjunta, sino de manera individualizada, eliminar las dudas que impregnan al paciente durante la consulta de enfermería o incluso en un diálogo informal entre el paciente y el profesional (DE OLIVEIRA et al., 2016).

Pero la educación para la salud no solo se identifica como un proceso de atención a los pacientes, las enfermeras también deben estar atentas a la capacitación y las calificaciones, que también pueden considerarse una educación para la salud de una manera más técnica. Silva; Santo y Chibante (2017) afirma que las enfermeras siempre deben estar actualizadas a las nuevas formas de cuidar los pies diabéticos, ya que los procesos de tratamiento se estudian cada vez más en la literatura científica y aportan innovaciones para la cicatrización o incluso la estadificación de heridas.

Por lo tanto, la educación para la salud, la educación continua y la educación continua son formas de atención que las enfermeras utilizan para mejorar el cuadro clínico de los pacientes con pies diabéticos. 


\subsection{TÉCNICAS DE EVALUACIÓN DEL PIE DIABÉTICO}

Los conocimientos técnico-científicos adquiridos durante las fases de educación de enfermería y en el contexto de la educación continua y permanente son factores relevantes para el tratamiento del pie diabético, por lo tanto, corresponde a la enfermera buscar conocimientos para realizar técnicas de evaluación seguras para pacientes con diabetes como método de prevención y para pacientes con pie diabético como medidas de prevención de complicaciones.

Arruda et al. (2019) afirma que el examen físico de los pies diabéticos son procedimientos realizados por enfermeras con el fin de evitar posibles complicaciones como: desarrollo de úlceras y amputaciones de los miembros inferiores. Por lo tanto, el desconocimiento de esta técnica induce las mayores tasas de complicaciones. Los autores mencionados señalan en otro momento que además de que la técnica no se utiliza debido a la falta de conocimiento de la enfermera, las condiciones de trabajo también influyen en el no desempeño de la técnica de evaluación, estas condiciones son referidas por los autores como infraestructura inadecuada y demanda de cuidados excesivos.

Vargas et al. (2017) trae en su estudio un contexto similar al estudio antes mencionado y agrega que las enfermeras necesitan más capacitación con respecto a las técnicas de evaluación relacionadas con el pie diabético, las enfermeras también informan que la gerencia no ofrece materiales para realizar estas técnicas y hablan sobre demandas excesivas.

Pereira et al. (2017) considera que se deben tomar acciones específicas para remodelar el sistema de atención a los pacientes con pie diabético, estas acciones deben estar relacionadas más allá del examen del pie, presentando una comunicación efectiva y educación para la salud.

Leal et al. (2016) considera que las enfermeras pueden tener desconocimiento del tema debido a varios factores y uno de ellos es señalado por los autores como la alta demanda de cuidados. Esta demanda implica el agotamiento físico y mental del 
profesional, lo que proporciona una falta de tiempo para la calificación profesional. En otro sentido, los autores mencionan que la demanda excesiva de un problema determinado proporciona al profesional hacer espacio para una educación sanitaria permanente, en la que este tipo de formación propone transformaciones en las prácticas profesionales basadas en problemas existentes en el proceso de su trabajo aprendiendo a reflejar la práctica dentro de su realidad.

La enfermería lleva consigo la función de tratar enfermedades de la piel y sus enfermedades adyacentes. Esto se debe a que las enfermeras tienen en sus atribuciones el cuidado del paciente y la necesidad de ampliar este cuidado asociado a enfermedades relacionadas con la piel (DA SILVA BRANDAO et al., 2016; SA et al., 2016).

\subsection{LA ENFERMERA MEDIADORA PARA EL AUTOCUIDADO DE LOS PACIENTES CON PIE DIABÉTICO}

Hablar de autocuidado se refiere a cualquier acción derivada del acto cotidiano del ser humano. En cuanto al autocuidado, Arruda et al. (2019) reportan que los pacientes con pie diabético tienden a generar un gran impacto económico, social, cultural, emocional y biológico, lo que favorece una mayor dependencia de los demás para ayudar en las actividades de la vida diaria y un impacto en su autocuidado y calidad de vida.

Scain; Franzen y Hirakata (2018) informan que el paciente debe ser consciente de la realización de sus propias actividades y que el autocuidado debe ser continuo, una de las formas de realizar el autocuidado citada por los autores fueron: Higiene de los pies; elección del calzado adecuado; evitar caminar descalzo y otros.

Vargas et al. (2017) trae como autocuidado el mantenimiento del control glucémico, la participación proactiva de los pacientes en la realización de exámenes de pies, terapia, restricción absoluta al consumo de tabaco y bebidas, nutrición, ejercicio físico, actividad física y otras formas que contemplan el estudio anteriormente referido. 
Una forma de lograr que el paciente se proponga el autocuidado la mencionan los autores Silva; Santo y Chibante (2017) como el fortalecimiento de los vínculos entre el paciente y el profesional de la salud. Este fortalecimiento favorece la seguridad del paciente y la credibilidad de que al realizar el autocuidado de los pies de forma continua y sistemática, las posibilidades de riesgo de complicaciones son menores.

La autonomía y seguridad que demuestra el paciente con pie diabético minimizan los daños y el riesgo de complicaciones. Un paciente que se involucra en el tratamiento de su enfermedad tiende a ampliar sus conocimientos para evitar la dependencia de otras personas, articulando el autocuidado en sus actividades como forma de motivación y demostración de capacidad frente a las adversidades que envuelven la salud-enfermedad (DE OLIVEIRA et al., 2016).

A Fátima Bento et al. (2016), los pacientes con pie diabético ya sufren estigmas por su condición, lo que les dificulta tener una atención adecuada, porque las condiciones que implican distanciarse de la unidad de salud, dificultades para movilizarse, poco conocimiento sobre la enfermedad, permiten una atención inadecuada y la evolución de las complicaciones.

Sin embargo, Nascimento et al. (2016) mencionan que cuando la atención calificada y humanizada se brinda a través de conductas efectivas, con fácil acceso a servicios de salud a los que se pueden integrar todas las acciones en todos los niveles de atención, el autocuidado sin riesgo de complicaciones.

\section{CONCLUSIÓN}

Este estudio reflexionó sobre el cuidado de enfermeras y profesionales en el desempeño de la atención y cuidado de pacientes con pie diabético. Los estudios de atención relevantes identificaron lo que llevó a la realización de tres temas para la discusión de los resultados encontrados.

El objetivo del estudio tuvo como objetivo resaltar la atención brindada a las enfermeras a los pacientes con pies diabéticos, se enfatiza que se enfatizó el 
cuidado de las enfermeras y no otras categorías de enfermería. Los estudios demostraron que la educación para la salud es la primera atención, lo que no puede ser contradicho, ya que la literatura enfatiza que la educación para la salud es primordial para la prevención de enfermedades.

Otro tipo de atención se refleja en el propio conocimiento del profesional sobre el pie diabético, de extrema necesidad y gran preocupación dentro del sistema de salud, ya que los artículos trajeron reflexiones de que las enfermeras deben estar más capacitadas para dirigir la atención con más excelencia al paciente.

Un cuidado importante también señalado por los estudios y que se dirige directamente con la formación permanente o continua de los profesionales son las técnicas de examen físico, en este tipo de cuidados, la enfermera debe ser capaz de realizar el examen de los pies diabéticos de forma inteligente y segura, además de también animar a los pacientes a acudir al examen con mayor frecuencia.

En cuanto a los cuidados relacionados con el autocuidado del propio paciente, se reflejó que la higiene de los pies, el control glucémico, el seguimiento continuo con el examen de los pies, el uso de calzado adecuado y otros intensifican una mayor prevención frente a complicaciones entre ellas la amputación de extremidades.

Se concluye, por lo tanto, que el estudio identificó varias formas de atención proporcionadas por las enfermeras y que todas estas formas son importantes para la continuidad del tratamiento y la prevención y las complicaciones, sin embargo, depende de la enfermera ser un profesional que valore la calidad de su atención en base a conceptos teóricos que conducen a la capacitación frecuente.

\section{REFERENCIAS}

AMERICAN DIABETES ASSOCIATION. Standards of medical care in diabetes. Diabetes Care, v. 40, n. suppl 1, p. s1-s128, 2017. 
ARRUDA, Cecilia et al. Tecnologia educativa para cuidados e prevenção do pé diabético/Educational technology for care and prevention of diabetic foot ulcers. Ciência, Cuidado e Saúde, v. 20, 2021.

ARRUDA, Luana Savana Nascimento de Sousa et al. Conhecimento do enfermeiro acerca dos cuidados com o pé diabético. Rev. enferm. UFPE on line, p. [1-8], 2019.

ASCHNER M, Pablo et al. Clinical practice guideline for the prevention, early detection, diagnosis, management and follow up of type 2 diabetes mellitus in adults. Colomb. Med., Cali, v. 47, n. 2, p. 109-130, junho. 2016.

CAMILLO, Bibiana Schultz et al. Ações de educação em saúde na atenção primária a gestantes e puérperas: revisão integrativa. Revista de enfermagem UFPE on line-ISSN: 1981-8963, v. 10, n. 6, p. 4894-4901, 2016.

CUBAS, Marcia Regina et al. Pé diabético: orientações e conhecimento sobre cuidados preventivos. Fisioterapia em movimento, v. 26, n. 3, 2017.

DE FÁTIMA BENTO, Leandra et al. A perspectiva da vulnerabilidade na avaliação do pé diabético sob a ótica de enfermeiros. Cogitare Enfermagem, v. 21, n. 1, 2016.

DE OLIVEIRA, Patrícia Simplício et al. Atuação dos enfermeiros da Estratégia Saúde da Família na prevenção do pé diabético Practice nurse family health strategy in the prevention of diabetic foot. Revista de Pesquisa Cuidado é Fundamental Online, v. 8, n. 3, p. 4841-4849, 2016.

DE SOUZA MENDONÇA, Sarah; MORAIS, Juliana de Sant'Anna; DE MOURA, Maria Catarina Gomes Gadelha. Proposta de um protocolo de avaliação fisioterapêutica para os pés de diabéticos. Fisioterapia em Movimento, v. 24, n. 2, 2017.

FELIX, Lidiany Galdino et al. Conhecimento de enfermeiros da atenção primária antes e após intervenção educativa sobre pé diabético. Revista Gaúcha de Enfermagem, v. 42, 2021. 
LEAL, Maria do Carmo et al. Atenção ao pré-natal e parto em mulheres usuárias do sistema público de saúde residentes na Amazônia Legal e no Nordeste, Brasil 2010. Rev. Bras. Saude Mater. Infant. Recife, v. 15, n. 1, p. 91-104, Mar. Available from \&lt; http://www.scielo.br/scielo.php?script=sci_arttext\&amp;pid=S151938292015000100091\&amp; Ing=en\&amp;nrm=iso\&gt;.

MENDES, Karina Dal Sasso; SILVEIRA, Renata Cristina de Campos Pereira; GALVÃO, Cristina Maria. Uso de gerenciador de referências bibliográficas na seleção dos estudos primários em revisão integrativa. Texto \& ContextoEnfermagem, v. 28, 2019.

MINAYO, Maria Cecília de Souza. Análise qualitativa: teoria, passos e fidedignidade. Ciência \& saúde coletiva, v. 17, p. 621-626, 2012.

MUZY, Jéssica et al. Prevalência de diabetes mellitus e suas complicações e caracterização das lacunas na atenção à saúde a partir da triangulação de pesquisas. Cadernos de Saúde Pública, v. 37, 2021.

NASCIMENTO, Vagner Ferreira do et al. Percepção de puérperas sobre as primeiras consultas de pré-natal no interior de Mato Grosso. Rev. enferm. UFPI, v.5, n. 1, p. 46-51, 2016.

ORGANIZAÇÃO MUNDIAL DE SAÚDE. Definition, diagnosis and classification of diabetes Mellitus and its complications. Geneva: OMS, 1999. Disponível em: https://apps.who.int/iris/bitstream/handle/10665/66040/WHO_NCD_NCS_99.2.pdf?s equence $=1$ \&isAllowed $=\mathrm{y}$

PEREIRA, Laiane de Fátima et al. Ações do enfermeiro na prevenção do pé diabético: o olhar da pessoa com diabetes mellitus. Rev. pesqui. cuid. fundam. (Online), p. 1008-1014, 2017.

SCAIN, Suzana Fiore; FRANZEN, Elenara; HIRAKATA, Vânia Naomi. Riscos associados à mortalidade em pacientes atendidos em um programa de prevenção do pé diabético. Revista Gaúcha de Enfermagem, v. 39, 2018. 
SILVA, Caline Oliveira da. Análise da qualidade de vida em indivíduos com Diabetes Mellitus Tipo 1. 2017. 34 f. Trabalho de conclusão de curso (Bacharelado em Farmácia) -Universidade de Brasília, Brasília, 2017. Disponível em: https://bdm.unb.br/handle/10483/19301

SILVA, Joziane Santos da; SANTO, Fátima Helena do Espírito; CHIBANTE, Carla Lube de Pinho. Alterações nos pés do idoso hospitalizado: um olhar cuidadoso da enfermagem. Escola Anna Nery, v. 21, 2017.

SOCIEDADE BRASILEIRA DE DIABETES. Tratamento e acompanhamento do diabetes Mellitus: Diretrizes da Sociedade Brasileira de Diabetes. 3.ed. Itapevi, SP: A. Araújo Silva Farmacêutica; 2017/2018. Disponível em: http://www.diabetes.org.br/ profissionais/images/2017/diretrizes/diretrizes-sbd-20172018.pdf.

VARGAS, Caroline Porcelis et al. Condutas dos enfermeiros da atenção primária no cuidado a pessoas com pé diabético. Rev. enferm. UFPE on line, p. 4535-4545, 2017.

Enviado: Octubre de 2021.

Aprobado: Diciembre de 2021. 